\title{
Comparative Analysis of the Complete Mitochondrion of Chlorophytum Comosum with 9 Other Species
}

\author{
Xueyu Cui \\ Nanning Normal University \\ Yujiao Peng \\ Nanning Normal University \\ Lin $\mathrm{Hu}$ \\ Nanning Normal University \\ Yuanyuan Shao \\ Nanning Normal University \\ Enling Song \\ Guangxi Subtropical Crops Research Institute \\ Yanlin Hou \\ Nanning Normal University \\ Shutian Liu ( $\nabla$ yaoyuan200452@163.com ) \\ Nanning Normal University
}

\section{Research Article}

Keywords: Chlorophytum comosum, Nanopore, Illumina, gene cluster, Selective Pressure

Posted Date: December 22nd, 2021

DOI: https://doi.org/10.21203/rs.3.rs-1068756/v1

License: () (i) This work is licensed under a Creative Commons Attribution 4.0 International License. Read Full License 


\section{Abstract}

Chlorophytum comosum belongs to genus Chlorophytum of family Asparagaceae that has more than 215 species. it is among 120 plant species assayed for phytoremediation of pollutants from indoor air. It has the ability to remove formaldehyde, nitrogen dioxide, carbon oxide, ozone, benzene, toluene, cigarette smoke and ammonia. It also is an important Chinese tradition medicine material. Aim to get more information about its mitochondrial genome, we sequenced the complete mitochondrial genome of C. comosum. The length of mitochondrial genome was 950316 bp with 36 protein coding genes, 5 rRNAs and 21 tRNAs. Although it has the biggest mitochondrial genome, the total PCGs content were the second least. C. comosum had the most repeats sequence and it may effect the size of mitochondrial genome, but base the result we found that the quantity of repeats sequence could not have the positive correlation with the mitochondrial size. Compared with other 9 species, we found in the mitochondrial genome of $\mathrm{C}$. comosum, there were at least one PCGs suffered from the positive selection after divergence. Finally, the result of phylogenetic showed that C. comosum did not have colesd relationship with other species.

\section{Introduction}

Chlorophytum comosum belongs to genus Chlorophytum and family Asparagaceae [1]. Genus Chlorophytum distributed in Africa (possibly the center of origin), Australia, Asia, Madagascar, and India and C. comosum mainly native to the tropics and southern Africa [2,3]. It has long and narrow leaves from the root. its leaf is green color or with white stripes on the edges and sometimes it also has white flowers [4]. It is known as indoor or outdoor ornamental plant, however, an important ability of $C$. comosum was remove hazardous in air such as formaldehyde, nitrogen dioxide, carbon oxide, ozone, benzene, toluene, cigarette smoke and ammonia [5-9]. Moreover, $C$. comosum have good accumulation on heavy metal in soil and easily removable, so it was widely used in interior design and urban microlandscape to improve the environment [10-13]. C. comosum is also well known as a kind of medicinal characteristics, it could be used to make steroidal and triterpenoidal saponin related medicines [14]. At same time, it is used to treat burns, bronchitis, fractures [15], and also has antitumor activity [16].

Mitochondria are complex intracellular organelles that have long been considered the power plants of cells because they play an important role in oxidative energy metabolism. Mitochondria, as metabolic centers, can rapidly adapt to different environmental signals and metabolic changes to meet the bioenergy requirements of cells, which has recently been defined as mitochondrial plasticity [17]. Mitochondria are highly plastic and a dynamic network of signaling organelles that play a multifunctional and critical role in cell metabolism, proliferation and survival[18]. Notably, various preclinical and clinical studies have shown that metabolic and/or inherited mitochondrial changes are involved in the pathogenesis of a large number of diseases, including cancer[19, 20].

Plant mitochondrial (mt) genomes are usually over 1000 times larger than those of animals, by frequent re-combination occurred they are structurally more complex [21]. The notably large size of plant $\mathrm{mt}$ genomes is shaped by a combination of several factors, including a rich gene set along with the abundant introns that carries, and the capability of uptaking and integrating intracellular transferred sequences from the chloroplast and nucleus [22, 23], and horizontally transferred genes from foreign donors [24, 25]. The studies about Chlorophytum comosum continued for long time, however, base the mt genomes of plants in genus Chlorophytum are fewer than other family. The data that we could find in NCBI about complete mitochondrial genome of family Asparagaceae was no more than 10 records. This state the study about this field could be woefully insufficient.

So this study, we will sequence the mitochondrial of Chlorophytum comosum and analysis (1) the characters of the mt genome, (2) comparative analysis the structure and of $\mathrm{mt}$ genomes with other species like codon usage, repeat sequence, gene cluster, Selective Pressure, (3) phylogenetic analysis.

\section{Methods}

\subsection{Plant materials}

In this study, the sample was collected from the flower market at 22 Yongwu Road, Chaoyang Street, Xixiangtang District, Nanning City, Guangxi province $\left(108^{\circ} 20^{\prime} 43^{\prime \prime} \mathrm{E}, 22^{\circ} 54^{\prime} 7 " \mathrm{~N}\right)$, the voucher specimen was stored in the Laboratory of the Ministry of Education, Nanning Normal University (specimen code NN202010). The study complies with relevant institutional, national, and international guidelines and legislation

\subsection{Sequencing, genome assembly and annotation}

We used the leaves of Chlorophytum comosum to extract total genome by using modified CTAB method[26]. Then we used Nanodrop(Nanodrop Technologies, Wilmington, DE, US), agarose gel electrophoresis and Qubit(Thermo Fisher Scientific,Waltham, MA, USA) to get the high quality DNA fragments. In sequencing step, we used both Illumina novaseq sequencing platform (Illumina, San Diego, CA, USA) and Nanopore PromethION sequencing platform(Nanodrop Technologies, Wilmington, DE, US). After using SQK-LSK109 to construct library. Large fragments with an average size of $10.72 \mathrm{~kb}$ were purified by magnetic bead enrichmen. Then $12.07 \mathrm{~Gb}$ raw data was sequenced by Nanopore PromethION sequencing platform. Then the raw data was filtered and reedited by NanoFilt and NanoPlot in Nanopack [27], and we got $11.93 \mathrm{~Gb}$ clean data with longest reads size156,720 bp. At the same time the high quality DNA fragments was constructed by using the NexteraXT DNA Library Preparation Kit. Then the libraries were sequenced on Illumina Novaseq 6000 platform and the average length of the generated read was $150 \mathrm{bp}$. The $5.19 \mathrm{~Gb}$ Illumina raw sequence read were edited using the NGS QC Tool Kit v2.3.3[28]. After using de novo assembler Canu2.1[29] and software Blastn[30] to assemble both Illumina and Nanopore clean data with the reference genome of Asparagus officinalis (MT483944), we got the mitochondrion contig with 950,316 bp length. Then we ran the correction step by BWA v0.7.12r103[31], and yielding a circular molecule of $950,316 \mathrm{bp}$. Following, we used Blastn and tRNAscan-SE 2.0[32, 33] to annotate the protein coding genes, rRNA 
genes and tRNA genes. Finally, OGDraw 1.2 was used to create gene clusters and the mitochondrial genome map[34]. The mitochondrial genome of $C$. comosum was submitted to NCBI under the assassin No.MW411187

2.3 Codon usage analysis, Selection pressure analysis and identification of repeat sequences

In codon usage analysis, we selected all protein coding genes from 10 MT genomes and then Synonymous codon usage and the relative synonymous codon usage (RSCU) was conducted to determine if the plastid genes were under selection by using codonW v1.4.4 [35]. Following step, we eliminated the stop codons and used Mafft v7.429[36] align the selected protein coding genes, and converted aligned files to PAML format. Nonsynonymous (Ka) and synonymous $(\mathrm{Ks})$ substitution ratio $(\mathrm{Ka} / \mathrm{Ks})$ of the mitochondrial PCGs of $C$. comosum were predicted using DnaSP v5.10.01 to identify the genes that are under selection pressure [37,38], finally PAML [39] was used to analyzed Ka/Ks with default setting. And repeat sequences was identified by software REPuter v2.74 with setting repeat sequence $\geqq 30$ bp and repeat identity $\geqq 90 \%[40]$

\subsection{Phylogenetic Analysis}

We selected the homologous genes that existed in all selected MT genomes and only had 1 copy in genomes. Then they were aligned by Mafft v.7.429 [36] and cleaned by Gblocks v.0.19b[41]. Using the model-finders[42] chops the model TIM+F+G4, then ML tree constructed by IQTree v1.6 [43].

\section{Results}

3.1 Mitochondrion structure and gene content.

Base the result, we found that the complete mitochondrial genome of Chlorophytum comosum had 950316 bp long with circle structure (Figure 1). The total GC content covered $46.65 \%$, Adenosine covered $26.62 \%$, Thymine covered $26.73 \%$, Cytosine covered $23.34 \%$ and Guanine covered $23.31 \%$. there were 62 genes in this mitochondrial genome with 36 protein coding genes, 5 rRNAs and 21 tRNAs. We also found the protein coding genes covered very small proportion in genome, the size only was $31500 \mathrm{bp}$ in total. In protein coding genes, GC content covered $43.66 \%$, Adenosine covered $26.54 \%$, Thymine covered $29.8 \%$, Cytosine covered $21.82 \%$ and Guanine covered $21.84 \%$. this was similar as the total GC content. Furthermore, base the result all protein coding genes only had one copy and 9 (nad7, nad1, nad4, ccmFC, rps10, rps3, nad2, nad5 and cox2) genes were containing one or more introns. For RNA, there were 2 rRNA genes ( $r r n 5$ and $r r n 8$ ) had 2 copies, and 7 tRNA genes (trnM-CAT, trnY-GTA, trnN-GTT, trnE-TTC, trnQ-TTG, trnH-GTG and trnW-CCA) had 2 or more copies especially trnM-CAT had 3 copies.

\subsection{Competitive analysis in Angiospermae}

Aim to acquire more information about the mitochondrial genome of $C$. comosum, we selected 9 complete mitochondrial genomes in Angiospermae from $\mathrm{NCBI}$ and compared with $C$. comosum by codon usage, repeat sequence, gene cluster, Selective Pressure and phylogenetic analysis.

\subsubsection{Codon usage analysis}

Base the result we found that there were 3 start codons and 3 stop codons. In start codons, ATG was the most frequent start codon that had been used in 32 protein coding genes in mitochondrial genome of $C$. comosum, then start codon ACG was used in 2 genes and start codon ATC was used in just 1 protein coding gene. In stop codons, stop codon TAA was the most frequent stop codon that had been used in 19 protein coding genes, then stop codon TGA was used in 10 genes and TAG was used in 7 genes. We calculated the relative synonymous codon usage (RSCU) to determine whether there was codon usage bias in coding sequences of $C$. comosum, and found that Methionine AUG and tryptophan UGG had no usage bias (RSCU $=1$ ), 30 codons were preferred codons (RSCU>1) (Figure 2).

In this study, we found another interesting thing that the quantity of codons was independent with the size of the mitochondrial genome. the largest mitochondrial genome was 950,316 bp and from C. comosum, but it did not contain the most codons, however, it just contained the second least codons (10500 codons) that meant the size of total protein coding genes was $31500 \mathrm{bp}$. And the most codons were contained in the mitochondrial genome of Cocos nucifera, the quantity was 18777 codons, however the size of this mitochondrial genome was $678,653 \mathrm{bp}$. But we also found that the smallest mitochondrial genome contained the least codons. it was Allium cepa and contained 8490 codons. The leaves of codons from the species in study were between 8490 and 18777, these meant that the range of the total size of protein coding genes would between 25470 and 56331 bp (Supplementary table S1).

Then we analysis the codon usage in 10 species, and found that the percentage of different codons were similar, the Leucine was the most amino acids around $10 \%$ in all of species in this study. Cysteine and Tryptophan were the least two amino acids in the species. In species Cocos nucifera, Lolium perenne and Phoenix dactylifera Tryptophan was the least amino acids and Cysteine was the least amino acids in others species around 1.3-1.67\% (Figure 3).

\subsubsection{Repeat sequence analysis}

Repeat sequence analysis have been widely used for species identification, genetic diversity studies, and evolutionary analysis, due to their high polymorphism level among the related species [44]. We detected repeat sequence of species in study. base the table 1 and figure 4 , we could find that 3833 repeats were detected in $C$. comosum that contained the most quantity of repeat sequence. 133 repeats sequence were detected in Phoenix dactylifera that 
contained the least quantity of repeats sequence. We divided these repeats sequence by the length, then we found that the sequence with the length $30-49$ bp would be the most repeats sequence in all species in this study. But the least repeats sequence was different among the species, such as in $C$. comosum the least repeats should be the length over $1000 \mathrm{bp}$, there were only 7 repeats, however in Allium cepa, there was no repeat with the size between 150 and $199 \mathrm{bp}$, so the least repeat sequence would be the repeat range around 150-199 bp.

Table 1 the repeat sequence in the mitochondrial genome of Chlorophytum comosum and other 9 higher plant mitochondrial genomes.

\begin{tabular}{|c|c|c|c|c|c|c|c|c|c|c|}
\hline & $\begin{array}{l}\text { Allium } \\
\text { cepa }\end{array}$ & $\begin{array}{l}\text { Bambusa } \\
\text { oldhamii }\end{array}$ & $\begin{array}{l}\text { Chlorophytum } \\
\text { comosum }\end{array}$ & $\begin{array}{l}\text { Cocos } \\
\text { nucifera }\end{array}$ & $\begin{array}{l}\text { Eleusine } \\
\text { indica }\end{array}$ & $\begin{array}{l}\text { Lolium } \\
\text { perenne }\end{array}$ & $\begin{array}{l}\text { Oryza } \\
\text { rufipogon }\end{array}$ & $\begin{array}{l}\text { Phoenix } \\
\text { dactylifera }\end{array}$ & $\begin{array}{l}\text { Tripsacum } \\
\text { dactyloides }\end{array}$ & $\begin{array}{l}\text { Zea } \\
\text { luxurians }\end{array}$ \\
\hline $30-49$ & 155 & 169 & 3436 & 91 & 96 & 111 & 188 & 89 & 1771 & 147 \\
\hline $50-69$ & 12 & 40 & 223 & 38 & 45 & 39 & 172 & 21 & 936 & 29 \\
\hline $70-99$ & 21 & 33 & 67 & 25 & 18 & 20 & 14 & 11 & 569 & 11 \\
\hline $\begin{array}{l}100- \\
149\end{array}$ & 4 & 8 & 30 & 12 & 7 & 14 & 14 & 5 & 229 & 1 \\
\hline $\begin{array}{l}150- \\
199\end{array}$ & 0 & 8 & 14 & 5 & 2 & 3 & 4 & 4 & 46 & 2 \\
\hline $\begin{array}{l}200- \\
999\end{array}$ & 1 & 21 & 56 & 13 & 6 & 20 & 3 & 2 & 6 & 7 \\
\hline$\geq 1000$ & 1 & 0 & 7 & 11 & 6 & 16 & 10 & 1 & 6 & 3 \\
\hline
\end{tabular}

Normally, the quantity of repeats may affect the size of genome, such as $C$. comosum has largest quantity of repeats sequence and the size of mitochondrial genome also ws the largest one. However, not all quantity had the positive correlation with the size of mitochondrial genome, such as Phoenix dactylifera, the mitochondrial genome of Phoenix dactylifera was the second largest mitochondrial genome, however it just had the least number of repeats sequences. Tripsacum dactyloides and Phoenix dactylifera had similar size around $700 \mathrm{~kb}$, but the number of repeats was huge different. $\mathrm{P}$. dactylifera only had 133 repeats but T. dactyloides has 3563 repeats.so it can hardly state that the repeats had the positive correlation with the size of mitochondrial genome.

\subsection{3 gene cluster analyses}

With the advancement of genomic sequencing and molecular biology, some similar clustered gene organization has been described in mitochondria of a number of green plants, from charophytic algae to angiosperms [45-48]. By the reason of the repeat mediated homologous recombination, sequence duplications, genome expansion and shrinkage, and incorporation of foreign DNAs, plant mitogenomes are highly fluid in genome structure [49]. in many instances, the ancestral ribosomal gene clusters are broken into much smaller ones containing no more than four genes [50,51]. However, in large scale phylogenetic, some gene clusters are still conserved [52]. But in most situation the gene organization greatly differs among plant mt genomes. In this study, we compared the gene orders in the $10 \mathrm{mt}$ genomes and counted the number of syntenic gene clusters (genes that remain in the same order). Gene clusters (rps12-nad3) was found to exist in all species mt genomes in this study (Figure 5, Supplementary Table S2). Gene cluster rrn18-rrn5 was absent in mt genome of Allium cepa, but existed in others. (nad5)-nad1-matR was lost in Oryza rufipogon, and rpl5-rps14 was only exist in species Chlorophytum comosum, Lolium perenne and Phoenix dactylifera, but absented or lost in others. It means these gene clusters would be highly conserved in plant mt genomes. The gene cluster matR-rpsl-ccmFN was absented in Phoenix dactylifera, Cocos nucifera, Chlorophytum comosum and Allium cepa but existed in other 6 species. However, the gene cluster atp4-nad4Lwas only exist in Allium cepa, Chlorophytum comosum, Cocos nucifera and Phoenix dactylifera.

Generally, the gene order and clusters would be the more similar by closer relationship in different species. it would be large different in gene order and clusters between different families, because genome recombination can disrupt clusters [53,54]. In this study, the selected species came from different family, but base the result we found gene clusters (rps12-nad3) was conserved plants mt genomes, and another two gene clusters (nad5-nad1-matR and rrn18-rrn5) conserved in most mt genomes (Table S2). These may reason that multiple recombination events can generate similar syntenic gene clusters, leading to vast differences in the gene order among plant mt genomes [53-56] In our study, the characteristics of the gene clusters in the Chlorophytum comosum mt genome were consistent with the general conservation of most monocotyledons, indicating that the gene clusters in the Chlorophytum comosum mt genome were more conserved during plant mt genome evolution.

\subsubsection{Selective Pressure Analysis}

One way to evaluate the selective pressures on protein evolution is to compare the rate of synonymous and non-synonymous nucleotide substitutions [57]. $\mathrm{Ks}$ is the estimated number of synonymous changes per synonymous site and corresponds to the rate of amino acid-neutral evolution. Ka, on the other hand, is the number of nonsynonymous substitutions per non-synonymous site. Under neutral protein- level evolution Ka should be equal to Ks and hence the ratio $\mathrm{Ka} / \mathrm{Ks}=1$ [58]. We compared 18 protein coding gene between Chlorophytum comosum and other species, then calculated the Ka/Ks. we found that most of $\mathrm{Ka} / \mathrm{Ks}$ value were under than 1 , at same time $19 \mathrm{Ka} / \mathrm{Ks}$ value were over 1 . The highest value of Ka/Ks was 2.115 that happened on gene 
rps14 compare with L. perenne. In addition, there were 10 genes that the Ka/Ks value $>1$, they happened on gene atp4, ccmC, nad2 compare with A. cepa, gene rpl5 compare with B. oldhamii, gene ccmB, mttB and nad2 compare with $\mathrm{C}$. nucifera, gene nad2 compare with $\mathrm{E}$. indica, gene mttB and rps14 compare with L. perenne, gene mttB, nad3 and rps1 compare with O. rufipogon, gene ccmB and nad2 complete with P. dactylifera, gene mttB and rps1 compare with $\mathrm{T}$. dactyloides, gene mttB compare with Z. luxurians. From the result, we could know that the most gene would be under stabilizing during evolution, but 10 genes would be suffered from the positive selection after divergence. (Figure 6)

\subsubsection{Phylogenetic analysis}

To confirm the phylogenetic position and understand the relationship of Chlorophytum comosum within Angiospermae. The complete mitochondrial genome of Allium cepa, Bambusa oldhamii, Eleusine indica, Tripsacum dactyloides Zea luxurians, Oryza rufipogon Lolium perenne, Cocos nucifera and Phoenix dactylifera were collected and aligned with Chlorophytum comosum, then got the result. The figure7 shows that Chlorophytum comosum did not have the very close relateship with other, however, we found Tripsacum dactyloides and Zea luxurians had close relationship, at the same time Cocos nucifera and Phoenix dactylifera also had close relationship. (Figure 4)

\section{Conclusion}

Through the result, we get the complete mitochondrial genome of Chlorophytum comosum with the length 950,316bp. It was the largest one in this study. There were 36 protein coding genes, 5 rRNAs and 21 tRNAs. Although it has the biggest mitochondrial genome, the total PCGs content were the second least. $C$. comosum had the most repeats sequence and it may affect the size of mitochondrial genome, but base the result we found that the quantity of repeats sequence could not have the positive correlation with the mitochondrial size. Compared with other 9 species, we found in the mitochondrial genome of $C$. comosum, there were at least one PCGs suffered from the positive selection after divergence. Finally, the result of phylogenetic showed that C. comosum did not have closed relationship with other species.

\section{Declarations}

Availability of data and materials: the raw sequence data of this study can be found in NCBI under Bio-project: PRJNA785105, and SRA Number: SRR17082800 and SRR17082801. The chloroplast genome sequence was under the accession number: MW411187 on NCBI website. Supplementary materials can be found at website

Consent for publication: the details/images/videos will be freely available on the internet and may be seen by the general public

Fundings: Guangxi Major Science and Technology Project (Guike AA17204077); Guangxi Construction Project of First-class Discipline (Geography)(2021); Open project Key Laboratory of Environmental Change and Resource Utilization of Beibu Gulf of Ministry of Education (GTEU-KLOP-X1820, GTEU-KLOP$\mathrm{X} 1819)$

Author Contributions: Xueyu Cui, Yujiao Peng, Lin Hu, Yuanyuan Shao, Enling Song, Yanlin Hou and Shutian Liu have equal Contributions

Acknowledgments: We sincerely thanks Total Genomics Solution Limited, SZHT. for performing the high throughput sequencing.

Conflicts of Interest: The authors declare no conflict of interest.

Ethics approval and consent to participate: Not applicable

\section{References}

[1] Chu C, Cui T, Li S, Zhan R, Gao Y. 2018. Structure and activity of a new sapogenin from Chlorophytum laxum R. Br. Chem Res Chin Univ. 34(5): 732-735.

[2] Govaerts, R.; Zonneveld, B.; Zona, S. World Checklist of Asparagaceae; Facilitated by the Royal Botanic Gardens: Kew, 2012.

[3] Malpure, N.V.; Yadav, S. Chlorophytum gothanense, a new species of Anthericaceae from the Western Ghats of India. Kew Bull. 2009, 64, 739-741.

[4] WFO (2021): Chlorophytum comosum (Thunb.) Jacques. Published on the Internet,http://www.worldfloraonline.org/taxon/wfo-0000763291. Accessed on: 13 Sep 2021

[5] Peart V (1992) Indoor air quality in Florida houseplants to fight pollution. Department of family, youth and community services, Florida cooperative extension service, institute of food and agricultural sciences. University of Florida, Gainesville, Publication FCS 3208

[6] Costa P, Eng C, James RW (1995) Constructive use of vegetation in office buildings. Proceedings the plants for people symposium, 23 XI 1995, Haga, Holandia, pp: 1-23

[7] Cornejo JJ, Ma CY, Stewart AJ (1999) Studies on the decontamination of air by plants. Ecotoxicology 8:311-320 
[8] Giese M, Bauer-Doranth U, Langebartels C, Sandermann H (1994) Detoxification of formaldehyde by the spider plant (Chlorophytum comosum) and by soybean (Glycine max L.) cell-suspension culture. Plant Physiol 104:1301-1309

[9] Wolverton BC (2008) How to grow fresh air-50 houseplants that purify your home or office. Cathy Meeus (ed) Weidenfeld \& Nicolson, pp: 8-27

[10] Soreanu G, Dixon M, Darlington A (2013) Botanical biofiltration of indoor gaseous pollutants-a mini-review. Chem Eng J 229:585-594.

[11] Gawrońska H, Bakera B (2015) Phytoremediation of particulate matter from indoor air by Chlorophytum comosum L. plants. Air Qual Atmos Health 8:265-272.

[12] Afton SE, Catron B, Caruso JA (2009) Elucidating the selenium and arsenic metabolic pathways following exposure to the nonhyperaccumulating Chlorophytum comosum, spider plant. J Exp Bot 60:1289-1297.

[13] Wang Y, Tao J, Dai J (2011) Lead tolerance and detoxification mechanism of Chlorophytum comosum. Afr J Biotechnol 10:14516-14521

[14] Kalra, S.; Kumar, S.; Lakhanpal, N.; Kaur, J.; Singh, K. Characterization of squalene synthase gene from Chlorophytum borivilianum (Sant. and Fernand.). Mol. Biotechnol. 2013, 54, 944-953.

[15] Kaushik, N. Saponins of Chlorophytum species. Phytochem. Rev. 2005, 4, 191-196.

[16] Haque, R.; Saha, S.; Bera, T. A peer reviewed literature on medicinal activity of Chlorophytum borivilianum commercial medicinal plant. Int. J. Drug Dev. Res. 2011, 3, 1116, 1130.

[17] Bahat, A.; Gross, A. Mitochondrial plasticity in cell fate regulation. J. Biol. Chem. 2019, 294, 13852-13863.

[18] Lisowski, P.; Kannan, P.; Mlody, B.; Prigione, A. Mitochondria and the dynamic control of stem cell homeostasis. EMBO Rep. 2018,19 , e45432.

[19] Wallace, D.C. A Mitochondrial Paradigm of Metabolic and Degenerative Diseases, Aging, and Cancer: A Dawn for Evolutionary Medicine. Annu. Rev. Genet. 2005, 39, 359-407.

[20] Niyazov, D.M.; Kahler, S.G.; Frye, R.E. Primary Mitochondrial Disease and Secondary Mitochondrial Dysfunction: Importance of Distinction for Diagnosis and Treatment. Mol. Syndr. 2016, 7, 122-137.

[21] Knoop V, Volkmar U, Hecht J, Grewe F. Mitochondrial genome evolution in the plant lineage. In: Kempken F. ed. Plant Mitochondria, Advances in Plant Biology 1. Springer Science + Business Media LCC; 2011. pp. 3-29.

[22] Alverson AJ, Wei X, Rice DW, Stern DB, Barry K, Palmer JD. Insights into the evolution of mitochondrial genome size from complete sequences of Citrullus lanatus and Cucurbita pepo (Cucurbitaceae). Mol Biol Evol. 2010; 27(6):1436-1448.

[23] Alverson AJ, Rice DW, Dickinson S, Barry K, Palmer JD. Origins and recombination of the bacterialsized multichromosomal mitochondrial genome of cucumber. Plant Cell. 2011; 23(7):2499-2513.

[24] Bock R. Witnessing genome evolution: experimental reconstruction of endosymbiotic and horizontalgene transfer. Annu Rev Genet. 2017;(51):1-22.

[25] Rice DW, Alverson AJ, Richardson AO, Young GJ, Sanchezpuerta MV, Munzinger J, et al. Horizontal transfer of entire genomes via mitochondrial fusion in the angiosperm Amborella. Science. 2013; 342 (6165):1468-1473.

[26] Healey, A.; Furtado, A.; Cooper, T.; Henry, R.J. Protocol: A simple method for extracting next-generation sequencing quality genomic DNA from recalcitrant plant species. Plant Methods 2014, 10, 21.

[27] Wouter De Coster, Svenn D’Hert, Darrin T Schultz, Marc Cruts, Christine Van Broeckhoven, 2018, NanoPack: visualizing and processing long-read sequencing data, Bioinformatics, Volume 34, Issue 15, Pages 2666-2669

[28] Patel RK, Jain M (2012) NGS QC Toolkit: A Toolkit for Quality Control of Next Generation Sequencing Data. PLoS ONE 7(2): e30619.

[29] Sergey, Koren, Brian, P. , Walenz, Konstantin, \& Berlin, et al. (2017). Canu: scalable and accurate long-read assembly via adaptive k-mer weighting and repeat separation. Genome research.

[30] Altschul, S.F. BLAST algorithm. e LS 2001.

[31] Li, H.; Durbin, R. Fast and accurate long-read alignment with Burrows-Wheeler transform. Bioinformatics 2010, 26, 589-595

[32] Chan, P.P.; Lowe, T.M. tRNAscan-SE: Searching for tRNA genes in genomic sequences. In Gene Prediction; Springer: Berlin, Germany, $2019 ;$ pp. 1-14.

[33] Lowe, T.M.; Chan, P.P. tRNAscan-SE On-line: Integrating search and context for analysis of transfer RNA genes. Nucleic Acids Res. 2016, 44, W54-W57. 
[34] Lohse, M.; Drechsel, O.; Kahlau, S.; Bock, R. OrganellarGenomeDRAW-a suite of tools for generating physical maps of plastid and mitochondrial genomes and visualizing expression data sets. Nucleic Acids Res. 2013, 41, W575-W581.

[35] CodonW, available online: http://codonw.sourceforge.net/, accessed on 19 December 2020

[36] Katoh, K.; Standley, D.M. MAFFT multiple sequence alignment software version 7: Improvements in performance and usability. Mol. Biol. Evol. 2013, $30,772-780$

[37] Librado, P.; Rozas, J. DnaSP v5: A software for comprehensive analysis of DNA polymorphism data. Bioinformatics 2009, 25, $1451-1452$.

[38] Rozas, J.; Sánchez-DelBarrio, J.C.; Messeguer, X.; Rozas, R. DnaSP, DNA polymorphism analyses by the coalescent and other methods. Bioinformatics $2003,19,2496-2497$.

[39] Yang, Z. 2007. PAML 4: a program package for phylogenetic analysis by maximum likelihood. Molecular Biology and Evolution 24: $1586-1591$

[40] Kurtz, S.; Choudhuri, J.V.; Ohlebusch, E.; Schleiermacher, C.; Stoye, J.; Giegerich, R. REPuter: The manifold applications of repeat analysis on a genomic scale. Nucleic Acids Res. 2001, 29, 4633-4642

[41] Talavera, G.; Castresana, J. Improvement of phylogenies after removing divergent and ambiguously aligned blocks from protein sequence alignments. Syst. Biol. 2007, 56, 564-577.

[42] Kalyaanamoorthy, S., Minh, B., Wong, T. et al. ModelFinder: fast model selection for accurate phylogenetic estimates. Nat Methods 14, 587-589 (2017).

[43] Nguyen LT, Schmidt HA, von Haeseler A, Minh BQ. IQ-TREE: a fast and effective stochastic algorithm for estimating maximum-likelihood phylogenies. Mol Biol Evol, 2015, 32(1):268-274

[44] Huang, J.C.; Sun, M. Genetic diversity and relationships of sweetpotato and its wild relatives in Ipomoea series Batatas (Convolvulaceae) as revealed by inter-simple sequence repeat (ISSR) and restriction analysis of chloroplast DNA. Theor. Appl. Genet. 2000, 100, 1050-1060.

[45] Regina TMR, Quagliariello C (2010) Lineage-specific group II intron gains and losses of the mitochondrial rps3 gene in gymnosperms. Plant Physiol Biochem 48:646-654

[46] Regina TMR, Picardi E, Lopez L, Pesole G, Quagliariello C (2005) A novel additional group II intron distinguishes the mitochondrial rps3 gene in gymnosperms. J Mol Evol 60:196-206

[47] Turmel M, Otis C, Lemieux C (2003) The mitochondrial genome of Chara vulgaris: insights into the mitochondrial DNA architecture of the last common ancestor of green algae and land plants. Plant Cell 15:1888-1903

[48] Richardson AO, Rice DW, Young GJ, Alverson AJ, Palmer JD (2013) The "fossilized" mitochondrial genome of Liriodendron tulipifera: ancestral gene content and order, ancestral editing sites, and extraordinarily low mutation rate. BMC Biol 11:29

[49] Palmer JD, Adams KL, Cho Y, Parkinson CL, Qiu YL, Song K. Dynamic evolution of plant mitochondrial genomes: mobile genes and introns and highly variable mutation rates. Proc Natl Acad Sci. 2000;97:6960-6.

[50] Grewe F, Viehoever P, Weisshaar B, Knoop V (2009) A trans-splicing group I intron and tRNA-hyperediting in the mitochondrial genome of the lycophyte Isoetes engelmannii. Nucleic Acids Res 37:5093-5104

[51] Terasawa K, Odahara M, Kabeya Y, Kikugawa T, Sekine Y, Fujiwara M, Sato N (2007) The mitochondrial genome of the moss Physcomitrella patens sheds new light on mitochondrial evolution in land plants. Mol Biol Evol 24:699-709

[52] Gui S, Wu Z, Zhang H, Zheng Y, Zhu Z, Liang D, Ding Y. The mitochondrial genome map of Nelumbo nucifera reveals ancient evolutionary features. Sci Rep. 2016; 6: 30158.

[53] Overbeek, R., Fonstein, M., D’Souza, M., Pusch, G. D. \& Maltsev, N. The use of gene clusters to infer functional coupling. Proc Natl Acad Sci USA 96, 2896-2901 (1999).

[54] Mower, J. P., Sloan, D. B. \& Alverson, A. J. Plant mitochondrial genome diversity: the genomics revolution (ed. Mower, P.) 123-144 (Springer Vienna, 2012)

[55] Bonavita, S. \& Regina, T. M. The evolutionary conservation ofrps3 introns and rps19-rps3-rpl16 gene cluster in Adiantum capillusveneris mitochondria. Curr Genet 62, 173-184 (2016).

[56] Dias, S. M., Siqueira, S. F., Lejeune, B. \& de Souza, A. P. Identification and characterization of the trnS/pseudo-trnA/nad3/rps12 gene cluster from Coix lacryma-jobi L: organization, transcription and RNA editing. Plant Sci 158, 97-105 (2000) 
[57] Robba, L.; Russell, S.J.; Barker, G.L.; Brodie, J. Assessing the use of the mitochondrial cox1 marker for use in DNA barcoding of red algae (Rhodophyta). Am. J. Bot. 2006, 93, 1101-1108.

[58] Song, H.; Chen, Y.; Gibson, K.; Liu, S.; Yu, Z.; Chen, N. High genetic diversity of the harmful algal bloom species Phaeocystis globosa revealed using the molecular marker COX1. Harmful Algae 2021, 107, 102065.

\section{Figures}

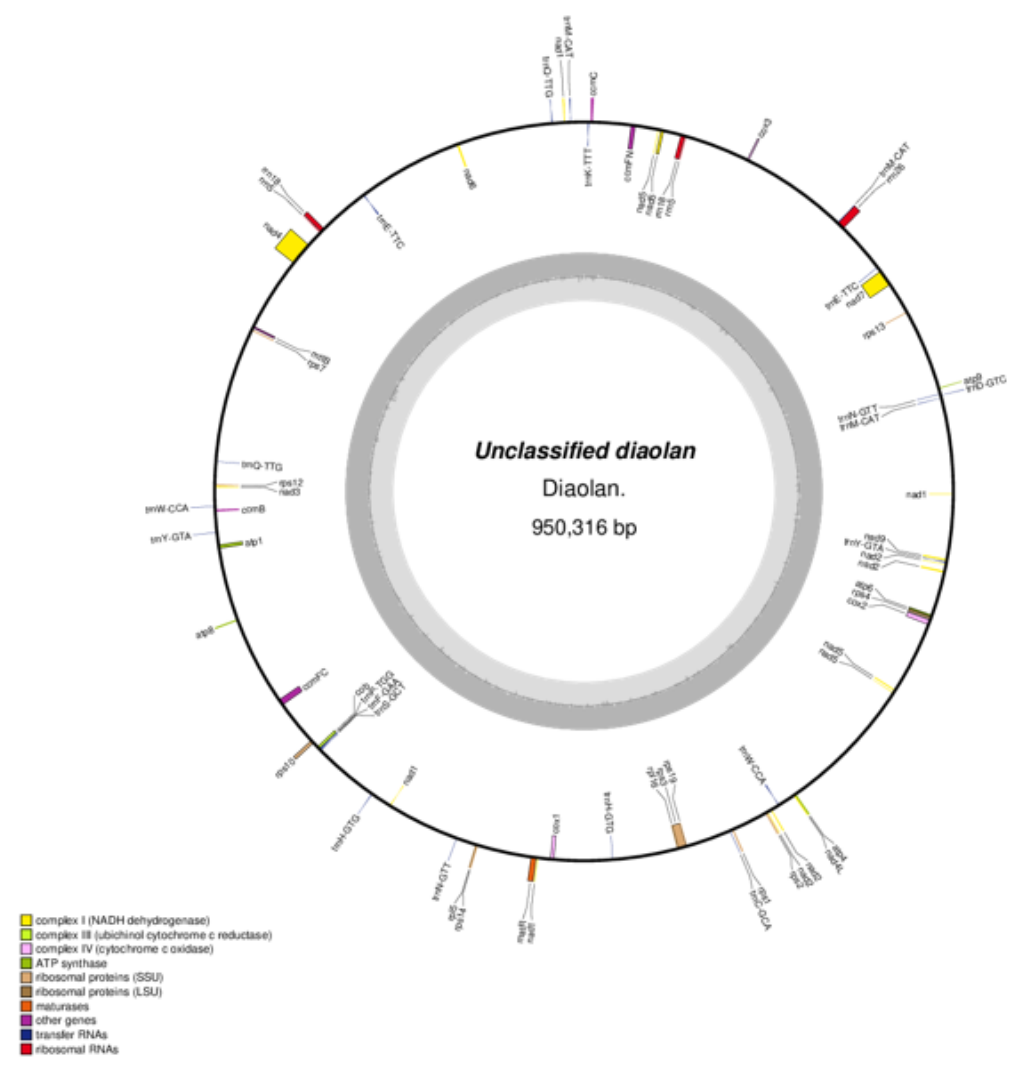

\section{Figure 1}

The structure of mitochondrial genome of Chlorophytum comosum 


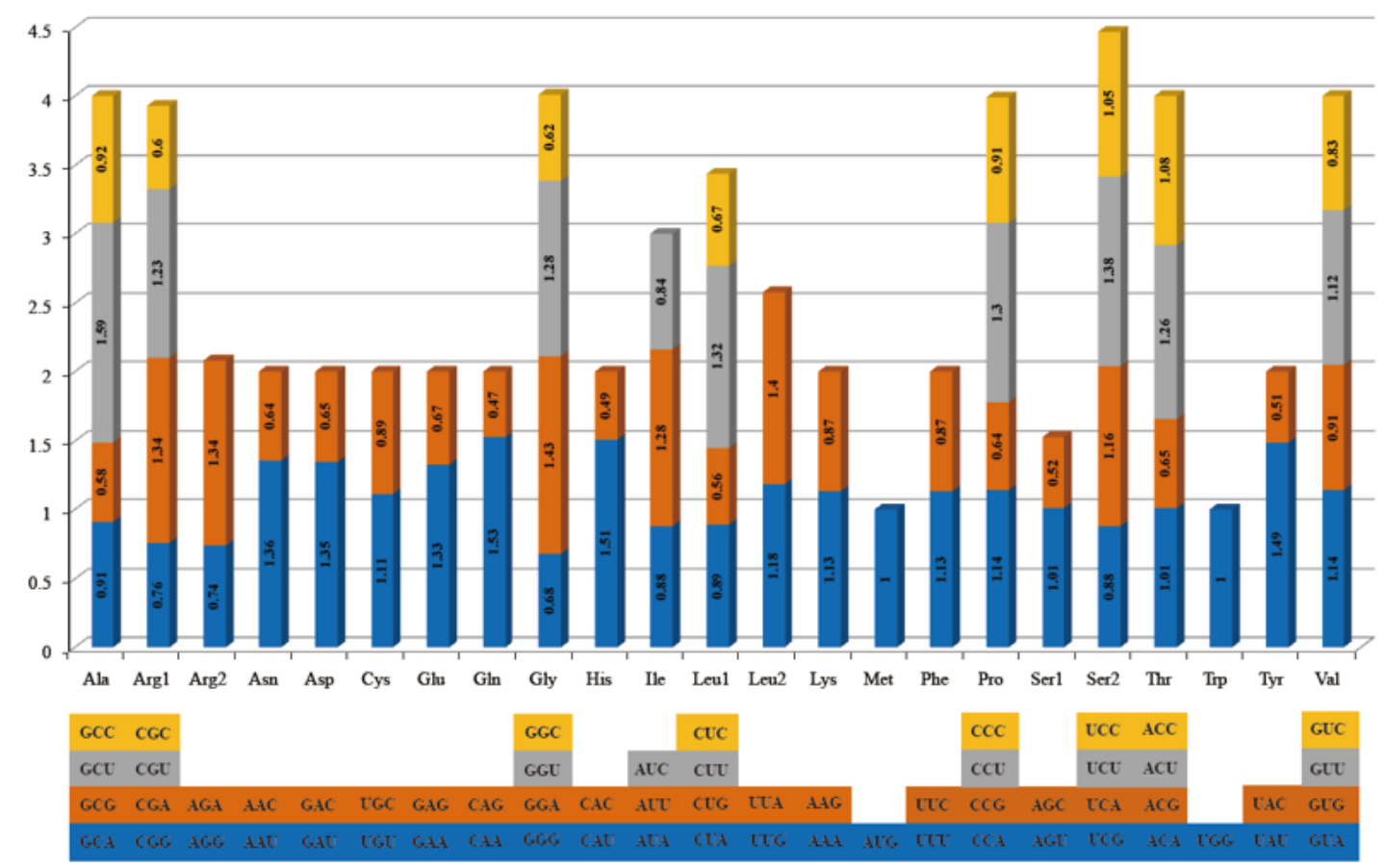

Figure 2

RSCU of mitochondrial genome of $C$. comosum

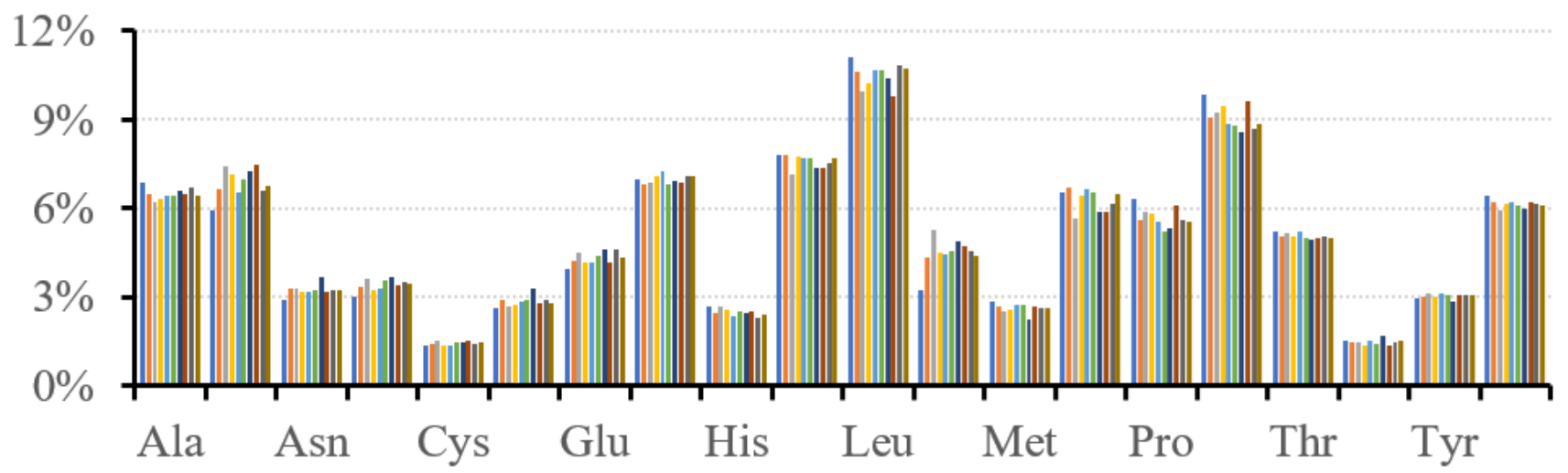

- Allium cepa

Chlorophytum comosum

- Oryza rufipogon

Zea luxurians
- Bambusa oldhamii

- Eleusine indica

- Phoenix dactylifera
- Cocos nucifera

- Lolium perenne

- Tripsacum dactyloides

Figure 3

codon usage of mitochondrial genome in 10 plants. 


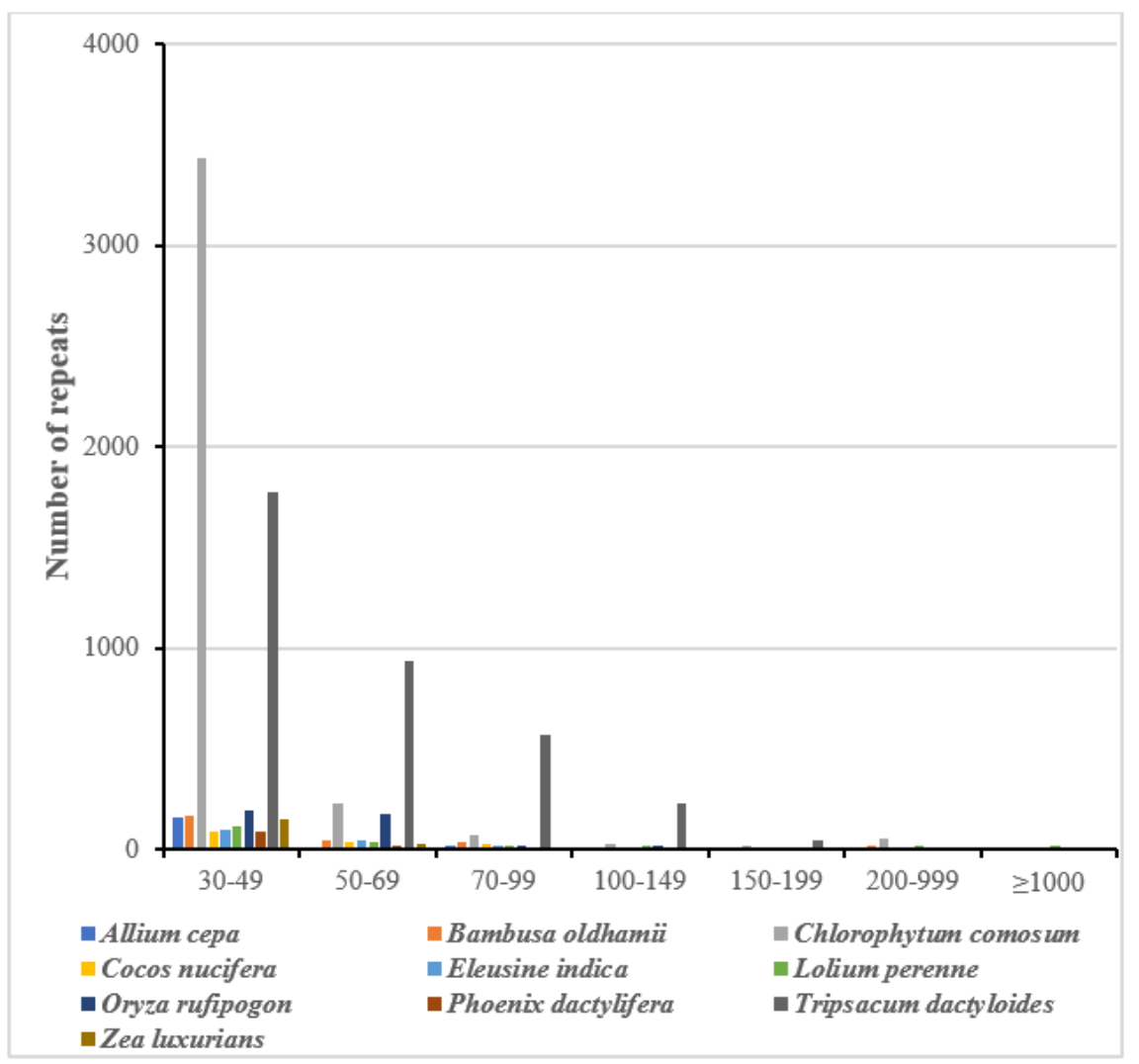

Figure 4

the repeat sequence in the mitochondrial genome in 10 plant mitochondrial genomes. 


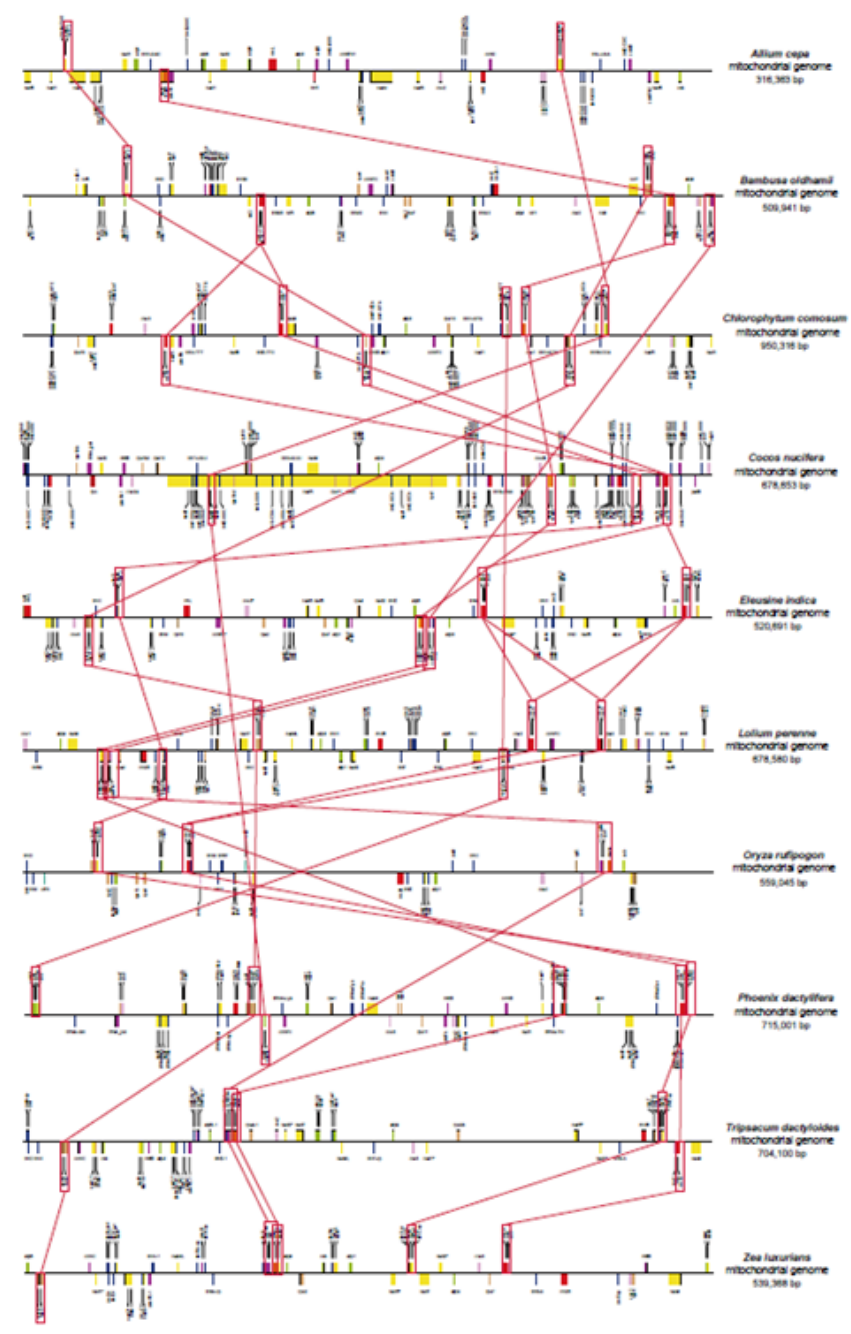

Figure 5

Analysis of conservative gene clusters between the Chlorophytum comosum mitochondrial genome and other higher plant mitochondrial genomes. 


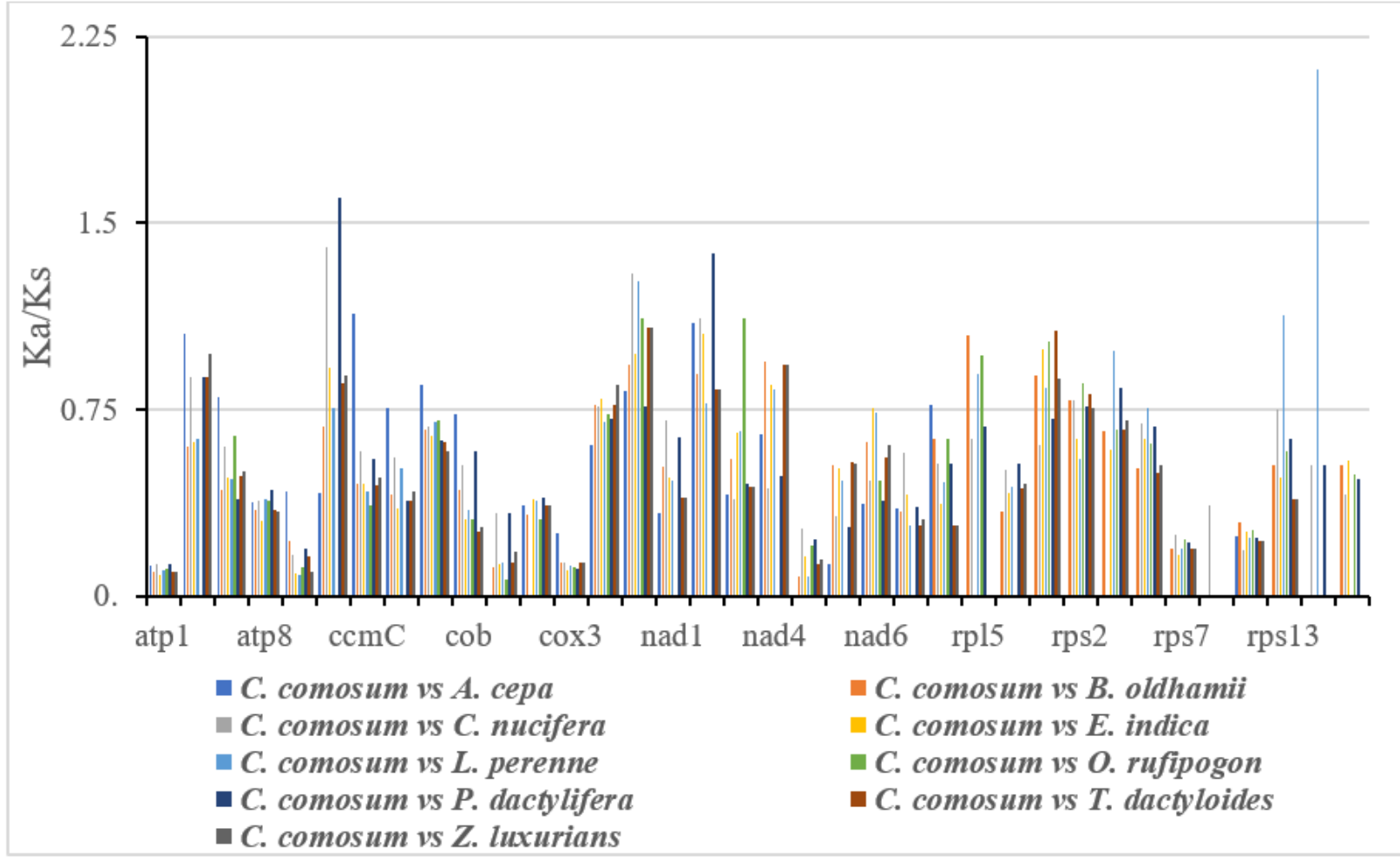

Figure 6

The value of $\mathrm{Ka} / \mathrm{Ks}$ of between Chlorophytum comosum and other nine species

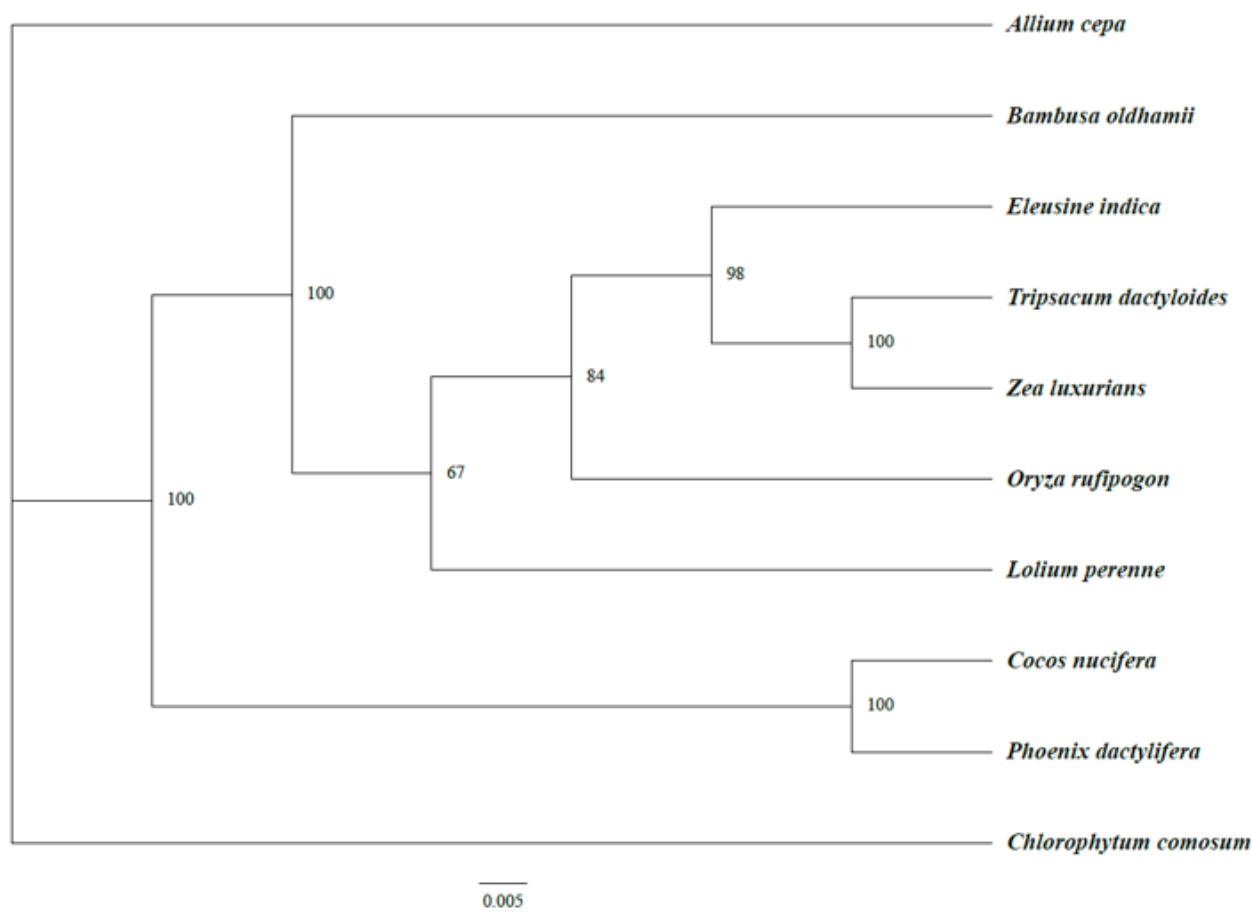

Figure 7

Maximum-likelihood phylogenetic tree for Chlorophytum comosum based on 10 complete mitochondrial genomes.

\section{Supplementary Files}

This is a list of supplementary files associated with this preprint. Click to download. 
- SupplementaryMaterial.zip

- tableS1thecodonsof10higherplantmitochondrialgenomes.xlsx

- TableS2AnalysisofconservativegeneclustersbetweentheChlorophytumcomosummitochondrialgenomeandotherhigherplantmitochondrialgenomes.xIsx 